\title{
Fatores humanos que influenciam a utilização de processos de software
}

\author{
Paula G. Ferreira ${ }^{1}$, Fabio Q. B. da Silva ${ }^{1}$ \\ ${ }^{1}$ Centro de Informática - Universidade Federal de Pernambuco (UFPE) \\ Caixa Postal 7851 - 50732-970 - Recife - PE - Brasil \\ \{pgf2, fabio\}@cin.ufpe.br
}

\begin{abstract}
Recent researches in software engineering indicate non-technical aspects as a factor that contributes to software project success and failure. Based on this premise, this paper presents a qualitative research conducted with quality area, project managers and leaders from software development projects, regarding the influence of team roles and personality in use of software development processes. The analyses show which psychological profile was more frequent on successful projects. Our aim is to contribute to the discussion of how teams could be balanced to increasing likelihood of process and project success.

Resumo. As pesquisas mais atuais na engenharia de software indicam o aspecto não técnico como um fator que contribui tanto para o sucesso quanto para o fracasso de projetos de software. Partindo dessa premissa, serão apresentados os resultados de uma pesquisa qualitativa realizada com a área de qualidade, gerentes e líderes de projetos de desenvolvimento de software, sobre a influência do papel de equipe bem como a personalidade na utilização de processos. As análises mostram qual perfil foi mais freqüente em processos com alto índice de aderência. Busca-se com esta pesquisa contribuir para a discussão sobre como montar equipes balanceadas e maximizar as chances de sucesso do processo e do projeto.
\end{abstract}

\section{Introdução}

A busca por implantação de processos de melhoria e certificações de qualidade tem aumentado no âmbito dos projetos de desenvolvimento de software das pequenas, médias e grandes empresas brasileiras. Cada vez mais são investidos recursos financeiros, técnicos e humanos para garantir produtos de qualidade, bem como projetos realizados no prazo, custo e qualidade definidos durante a fase de planejamento dos projetos [Vasconcelos et al. 2006].

Algumas empresas e consultorias relataram suas experiências, pontos fortes e fracos, desafios e riscos da implantação de um processo de software [Souza et al. 2004, Rocha et al. 2005, Roullier et al. 2006, Mendes et al. 2007]. Os dados mostram que além dos problemas de alto custo e falta de apoio da alta gerência, destaca-se também a barreira da cultura organizacional e falta de envolvimento e comprometimento dos envolvidos entre os motivos de insucesso do processo.

A pesquisa de Sharp e Robinson (2005) apresenta aspectos sociais que afetam a 
engenharia de software que englobam aspectos provenientes das várias interações interpessoais necessárias para o desenvolvimento do software, desde a equipe ao cliente. Segundo estes autores, a engenharia de software é muito mais uma atividade social com atividades técnicas do que uma atividade técnica com fatores sociais. Entretanto, ainda são escassas as pesquisas sobre este assunto que tenham como contexto a realidade brasileira em relação à utilização de processos de software.

Diante desses desafios, o foco deste trabalho está no elemento humano, mais especificamente no que tange o comportamento em equipe e a personalidade dos gerentes e líderes envolvidos em projetos que utilizam processos de desenvolvimento de software aderentes ao CMMI nível 3, e como eles influenciam e motivam a equipe a utilizar os processos. A hipótese deste trabalho é que existe uma tendência natural de pessoas com determinados perfis a se interessarem pela área de qualidade e pelo uso do processo.

A metodologia do trabalho consiste na avaliação qualitativa (através dos métodos SPI e MBTI) do padrão de papéis de equipe e tipos psicológicos identificados na área de qualidade, e a correlação dos mesmos com o uso do processo (avaliado através das auditorias de processo). Os dados foram coletados em um extenso trabalho de campo, realizado durante o ano de 2007 em uma empresa de software de grande porte do Nordeste do Brasil.

Este trabalho está organizado da seguinte forma. Na Seção 2 estão apresentados os intrumentos de pesquisa utilizados e os trabalhos relacionados. Na seção 3 será explicada a metodologia. Na seção 4 serão apresentados os dados da pesquisa de campo e os resultados obtidos. Por fim, na seção 5, serão apresentadas as conclusões.

\section{Instrumentos e Trabalhos Relacionados}

A engenharia de software possui poucos trabalhos que relacionam tipo de personalidade e efetividade do uso de processos de desenvolvimento de software. Alguns trabalhos apresentam resultados sobre o relacionamento entre os tipos de personalidade com papéis funcionais do RUP (Rational Unified Process) [França e Silva 2005], ou com modelos como PMCD (Project Management Competency Development) [Fernandes e Silva 2007], ou ainda com as práticas definidas no PMBoK (Project Management Body of Knowledge) [Pereira 2005].

Outras pesquisas relacionam o tipo de personalidade ao desempenho de atividades específicas [Cunha and Greathead 2007], ou a influência de um determinado perfil na composição da equipe [Stevens 1998; Henry and Stevens 1999; Gorla and Lam 2004].

Tendo em vista que pesquisas mais recentes mostram a importância de relacionar o trabalho em equipe e a personalidade em relação às atividades de gerência de projeto, assim como o desempenho em equipes e atividades de revisão de código, esta pesquisa visa identificar a relação entre comportamento em equipe e personalidade e sua influência na utilização de processos de desenvolvimento de software em uma empresa do Nordeste do Brasil. 
Busca-se identificar quem apóia o uso do processo de forma a permitir que equipes sejam formadas a partir desse conhecimento, aumentando as chances de uso do processo no projeto e, conseqüentemente, o sucesso do projeto. Para tanto, foram utilizados os instrumentos Self-Perception Inventory (SPI) e o Myers-Briggs Type Indicator (MBTI) e os trabalhos relacionados às atividades de engenharia de software.

\subsection{Os papéis de equipe de Belbin}

Em 1981, o doutor Meredith Belbin lançou um livro sobre gerenciamento de equipes baseado na teoria de papéis que ele desenvolveu. O livro surgiu de um experimento acadêmico realizado durante 9 anos no Henley Management College. Os padrões de comportamento identificados por Belbin foram classificados e nomeados em oito tipos de papéis de equipe.

A lista abaixo apresenta os oito papéis de equipe sugeridos por Belbin:

- Co-ordinator (CO) : organiza, coordena e controla as atividades da equipe. Isto envolve esclarecer objetivos e problemas. Atribui tarefas e responsabilidades, e incentiva os membros a se envolverem para atingir os objetivos e metas;

- Shaper (SH) : desafia, argumenta e discorda. Motivado a realizações, extrovertido, impaciente, tende a se frustar. Interesse em ganhar o jogo. Tem boa visão, especialmente se perde;

- Plant (PL) : concentrado em apresentar idéias e estratégias para atingir os objetivos da equipe. Este papel requer criatividade, imaginação e inovação;

- Resource Investigator (RI) : explora o ambiente fora do time, identificando idéias, informações e recursos. Desenvolve bem os contatos de coordenação e negociação com outros times e indivíduos;

- Monitor Evaluator (ME) : analisa idéias e propostas da equipe, avalia a viabilidade e valor para aintigir os objetivos. Considera de forma construtiva as debilidades das propostas;

- Completer Finisher (CF) : garante que o esforço da equipe atinge os padrões e que os erros sejam evitados. Busca por detalhes e mantém o senso de urgência da equipe;

- Team Worker (TW) : cria e mantém o espírito de equipe. Promove a comunicação através de apoio pessoal e cordialidade entre os membros, e evita tensões e conflitos;

- Implementer (IM) : preocupado que a aplicação de conceitos e planos sejam devenvolvidos pela equipe. Isto envolve perserverança face às dificuldades.

Em 1993, Belbin indicou mais um papel chamado de Specialist, mas muitos autores criticam sua necessidade, e a maior parte dos trabalhos ainda utiliza o modelo de 8 papéis, e por isso também foi utilizado nesta pesquisa.

Belbin defende que estes papéis quando colocados juntos numa mesma equipe, garantem uma coesão e sintonia entre os membros. Uma equipe não pode ser formada apenas por pessoas extrovertidas, como também não pode existir apenas pessoas 
coordenadoras. Deve haver um equilíbrio entre as forças e fraquezas da equipe.

O questionário ficou conhecido como Self Perception Inventory (SPI) e foi bastante difundido na Europa pela sua facilidade, rapidez e lógica de utilização. O modelo já foi traduzido para 16 línguas e tem sido utilizado por diversas organizações incluindo o grupo de companhias do FTSE 100 (Financial Times Stock Exchange) que representam 80\% do mercado do Reino Unido, agências multinacionais, governo e consultorias [Aritzeta; Swailles; Senior 2005].

É importante ressaltar que uma pessoa pode apresentar todos os 8 papéis do SPI em maior ou menor escala. Eles são agrupados em muito alto, alto, moderado e leve. Para este trabalho foram considerados os papéis das categorias muito alto e alto.

\subsubsection{Uso do SPI}

A pesquisa de Stevens (1998) apresentou resultados positivos ao utilizar o questionário de Belbin e comprovar empiricamente a eficácia da formação de equipes balanceadas com os papéis de equipe sugeridos por Belbin. Foi avaliado apenas o papel inovador do SPI e o impacto da sua presença no desempenho das equipes, visto que em equipes de desenvolvimento, o papel de inovação é fundamental, pois traz novas idéias à equipe, principalmente na fase inicial do desenvolvimento.

Em outra pesquisa, Henry e Stevens identificaram que a presença de um dos papéis de liderança de Belbin faz diferença no desempenho da equipe [Henry and Stevens 1999]. Eles realizaram um experimento controlado com estudantes seniors de engenharia de software, no qual os estudantes foram agrupados em equipes com apenas um líder e equipes com nenhum ou mais de um líder, nesse segundo caso houve conflito de liderança.

\subsection{Os tipos psicológicos do MBTI}

O MBTI [Myers and Briggs 2007] foi criado em 1942 por Isabel Briggs Myers e Katherine Cook Briggs. Elas desenvolveram o instrumento de indicador de personalidade após dezoito anos de estudos e aplicação da teoria Junguiana, cujo objetivo foi medir traços como inteligência e definir as preferências de personalidade.

O instrumento foi publicado pela primeira vez em 1962 e usado para fins de pesquisa. Em 1975 o MBTI passou a ser largamente difundido e disponibilizado para o mercado através do Consulting Psychologists Press. No Brasil sua aplicação é administrada exclusivamente pela Coaching Psicologia Estratégica, que realiza seminários de qualificação para profissionais.

No MBTI, as preferências de personalidade são agrupadas em quatro grandes aspectos: como a pessoa interage com o mundo e obtém energia (Extrovertida/Introvertida), como a pessoa percebe e assimila as informações (Sensitiva/Intuitiva), como a pessoa toma decisões e chega a conclusões (Pensamento /Sentimento), e como a pessoa organiza sua vida (Julgamento/Percepção). Os tipos psicológicos citados por Myers-Briggs são classificados nestas quatro dimensões, cuja escala de preferência varia entre dois extremos. 
O MBTI pressupõe que as pessoas utilizam vários processos cognitivos, mas tendem a ter uma preferência entre as dicotomias citadas. Cada tipo tem qualidades, riquezas e possibilidades de desenvolvimento. Esses quatro pares de combinação resultam em dezesseis tipos de personalidade.

Como resultado dessas funções surgiu a classificação dos tipos através de letras emprestadas de palavras inglesas cujas iniciais são: Extraversion/Introversion, Sensing/iNtuition, Thinking/Feeling , Judging/Perceiving. As letras iniciais de cada par de atributos, exceto iNtuition que usa a segunda letra, são combinadas e identificam a personalidade da pessoa, resultando em um código de quatro letras.

A lista abaixo apresenta a descrição das características de cada um dos 16 tipos:

- ISTJ - Responsável, cumpre os compromissos. Tipo o gerente essencial. Trabalha duro mas se diverte de fato nas horas de folga. Vive para os resultados e pode ser muito preocupado com custos;

- ISFJ - Sério em relação aos compromissos, responsável e leal. Leva o trabalho a sério, é dedicado e cumpre o dever sob todos os aspectos da vida. Quieto, fica contente em trabalhar sozinho. Está sempre ajudando no trabalho de outros;

- ISTP - É freqüentemente mal entendido e subestimado. É o tipo solitário que prefere fazer a planejar. Produz resultados sem se atrapalhar com entraves burocráticos. Prefere apagar incêndios a treinar formas de prevenção dos mesmos;

- ISFP - O tipo de pessoa que recusa uma promoção para poder continuar onde as coisas acontecem. Viver e deixar viver é a sua filosofia e o trabalho é a chave de sua motivação. Apóia e ajuda todos os colegas de trabalho;

- ESTP - Gosta de enfrentar riscos, experimentar e é empreendedor. É um tipo com várias aptidões. "Fazedor" com uma grande energia que o mantém envolvido em diversos projetos, animando todos e fazendo com que a vida seja excitante;

- ESFP - Corajoso e não-conformista. Se o trabalho não for divertido, irá evitá-lo ou mudar para algo mais interessante. Muito sociável, gosta de interagir com alegria e energia. Seu ponto forte está em permitir que outros sejam diferentes e trabalhem à sua maneira;

- ESTJ - Responsável, produtivo e orientado para resultados. Encontrado em posições de liderança em várias profissões. Quando um trabalho precisa ser feito, normas estabelecidas, sistemas implementados ou um programa avaliado chame o ESTJ;

- ESFJ - Elegância. Define o seu estilo de vida e gerencial. Encoraja e motiva as pessoas a atingirem seus objetivos. Gosta de um ambiente de trabalho formal, mas agradável. Possui uma tendência para agir paternalmente e, às vezes, com alguma impaciência. Distribui recompensas e também críticas. Bom fornecedor de feedbacks;

- INFJ - Estudioso, confiável no trabalho. Um tipo popular na indústria de serviços porque sempre dirige a sua energia e atenção para melhorar a condição 
humana. Uma pessoa gentil que se importa genuinamente com os outros. Gosta de ordem e tranqüilidade no ambiente de trabalho. Intelectual e idealista;

- INTJ - Possui visão e capacidade para torná-la realidade. Habilidades de liderança fazem com que desempenhem um papel muito importante na formação da cultura das corporações. Independência é a força que os motiva. É perfeito para o papel de especialista de "solucionador de problemas";

- INFP - Mais produtivo na área de serviços ou para trabalhar com idéias. Não tolera rotina. Precisa trabalhar de acordo com seus valores pessoais. Seu trabalho é voltado para pessoas. Tende a tomar decisões subjetivamente, baseando-se em valores pessoais, não gosta de controlar as pessoas. Sente-se confortável com a filosofia do "viver e deixar viver";

- INTP - Professores e pensadores. Ama o abstrato e precisa ficar sozinho para pensar e repensar suas idéias. Ele estuda e pesquisa profundamente qualquer assunto ou trabalho pelo qual seja responsável. Fonte de idéias e inspiração, mas não se preocupa com prazos no trabalho. Possui pensamento claro e visão na execução de seus projetos;

- ENFP - Este tipo desempenha muito bem num cargo executivo embora suas características de efervescência, entusiasmo e espontaneidade não sejam típicas nos ocupantes de altos cargos executivos nas empresas. Pode ser muito habilidoso em situações de improviso e pode fazer várias coisas ao mesmo tempo, embora, às vezes, negligencie o planejamento e a preparação;

- ENTP - Nunca se sabe o que esperar. Todo o momento é bom para alguma coisa e suas características são: energia, dinamismo, criatividade, resistência e argumentação. Prefere se envolver em desafios intelectuais a fazer um trabalho rotineiro. Gosta de público e tende a perder o interesse pelo que está fazendo se alguma coisa mais excitante ou desafiante aparecer;

- ENFJ - É o vendedor nato com grande poder de persuasão. Prefere viver sua vida de forma estruturada e ordeira. Muito atento à dinâmica interpessoal de cada situação, sente necessidade de liderar;

- ENTJ - Possui a mistura certa das qualidades de liderança: entusiasmo, visão, objetividade e responsabilidade. O ENTJ é forte, direto e bom estrategista, pode ver possibilidades em quase tudo e agir rapidamente.

\subsubsection{Uso do MBTI}

A pesquisa de Gorla e Lam (2004) teve como objetivo identificar a relação entre a composição dos tipos psicológicos e o desempenho em pequenas equipes de software de Hong Kong. Como resultado foram identificadas algumas caracterísitcas do líder de equipe, do analista e do programador que afetavam o desempenho da equipe. Por exemplo: um líder de equipe intuitivo $(\mathrm{N})$ e sentimental $(\mathrm{F})$ funcionava bem em equipes pequenas.

No caso do analista, foi identificado que o tipo pensador $(\mathrm{T})$ teve mais influência, pois nessa atividade as habilidades analíticas são mais importantes do que as comportamentais. Em equipes pequenas, o analista não foca apenas nos requisitos e 
especificação do sistema, ele está envolvido em múltiplas atividades que necessitam de uma abordagem científica e decisões lógicas.

Para o programador a forte influência foi apresentada no aspecto social, sendo o tipo extrovertido (E) mais significativo para equipes pequenas. Isto se explica pelo fato do programador ter que se comunicar com muitas pessoas, analistas, designer e outros programadores. $\mathrm{O}$ autor conclui que é diferente alocar pessoas em equipes pequenas $\mathrm{e}$ grandes, e por isso o gerente deve ficar atento e considerar a heterogeneidade no momento de selecionar sua equipe.

A pesquisa de Cunha e Greathead (2007) teve como objetivo investigar se existia um tipo psicológico relacionado com o desempenho na atividade de revisão de código. Os autores detectaram que alguns profissionais apresentaram mais habilidade do que outros. De uma forma geral, as pessoas com perfil NT tiveram melhor perfomance dos que os nãoNT. A maior diferença foi entre as pessoas com o tipo NT e SF. Os NTs são conhecidos como "lógicos e habilidosos" e são melhores na resolução de problemas, por isso não foi uma surpresa que este tipo fosse melhor nesta atividade. Entretanto, a grande diferença entre NT e SF, que são tipos opostos, foi que chamou a atenção, pois foi muito grande: os SF acertaram menos do que a metade do que os NT.

\section{Metodologia}

$\mathrm{O}$ arcabouço metodológico envolveu a seleção de projetos de software que utilizam processos aderentes ao modelo CMMI nível 3 como guia para o desenvolvimento do projeto. A empresa na qual foi realizada a pesquisa teve o seu processo estabelecido desde 1996, e vêm amadurecendo ao longo dos últimos 11 anos, e recentemente, em Outubro de 2007, obteve a certificação CMMI nível 3.

Foram analisados 9 projetos diversificados em seus domínios, prazos, tamanho das equipes, papéis de equipe e tipos psicológicos envolvendo 37 pessoas, das quais 15 da área de qualidade e 22 gerentes e líderes de projetos. A pesquisa teve abordagem qualitativa com a aplicação de questionários para identificação dos perfis, e foram analisados os resultados das auditorias de processos de planejamento e acompanhamento desses projetos.

Para classificação dos papéis de equipe e tipos psicológicos foram utilizadas as ferramentas SPI e o MBTI. Os percentuais de aderência dos processos de planejamento (Project Planning - PP) e acompanhamento (Project Monitoring and Control - PMC) foram analisados e relacionados aos papéis exercidos pelos gerentes e líderes.

O objetivo foi avaliar qual o padrão de papéis da área de qualidade, e qual a relação entre os papéis dos gerentes e líderes na utilização de processos nos projetos. Quanto maior o percentual atingido, maior a possibilidade de adequação do papel ao uso do processo. $\mathrm{O}$ resultado foi avaliado em relação aos papéis identificados na área de qualidade, visto que pessoas que atuam nesta área são consideradas favoráveis à utilização de processos.

Para este trabalho foi considerada a premissa de que o uso do processo depende da disposição das pessoas em utilizar e melhorar o processo. Não é possível avaliar, julgar ou 
criticar um processo se o mesmo não for utilizado. Independente do modelo, existem pessoas que vão utilizar e contribuir para a melhoria do processo, e existem pessoas que não vão se adaptar a seguir padrões, procedimentos, nem ao menos contribuir através de críticas. A hipótese é que isto depende do perfil de cada pessoa.

\subsection{Caracterização da Amostra}

Os projetos analisados foram classificados quanto à duração e tamanho da equipe conforme tabela 1:

Tabela 1: Duração e tamanho da equipe

\begin{tabular}{|c|c|c|}
\hline Tamanho & Grande (G) & Maior que 8 meses \\
\hline \multirow[t]{2}{*}{ Projeto (Tempo) } & Médio (M) & Entre 4 e 8 meses \\
\hline & Pequeno $(\mathrm{P})$ & Até 4 meses \\
\hline \multirow{3}{*}{$\begin{array}{l}\text { Tamanho da } \\
\text { Equipe }\end{array}$} & Grande (G) & Mais que 30 pessoas \\
\hline & Média (M) & Entre 11 e 30 pessoas \\
\hline & Pequena $(\mathrm{P})$ & Até 10 pessoas \\
\hline
\end{tabular}

Para efeito de anáise, a classificação dos projetos em relação ao uso do processo foi dividida em 3 grupos:

- Satisfatório (S): considera os projetos que contêm uma aderência ao processo igual ou superior a $90 \%$;

- Adequado (A): considera os projetos que contêm uma aderência ao entre $40 \%$ e $89 \%$;

- Insatisfatório (I): considera todos os projetos abaixo de $39 \%$.

\subsection{Limitações}

Foi realizado um levantamento dos projetos dentro da organização, e foram selecionados todos que utilizavam o processo aderente ao modelo CMMI nível 3 (18 no total). Entretanto, para esta pesquisa, foram removidos os projetos que se diferenciavam no tipo, por exemplo: consultoria e desenvolvimento. $\mathrm{O}$ foco foi nos projetos de desenvolvimento.

Outros projetos foram retirados da pesquisa por apresentarem dados insuficientes dos participantes (gerentes e líderes) ou por serem de caráter confidencial. Alguns projetos também foram removidos, quando foi identificado, previamente, que a causa do problema do projeto foi proveniente de outras fontes, como falta de experiência do gerente, e por isso, a utilização do processo não pode ser avaliada. Desta forma, foram selecionados apenas os projetos cujas variáveis tipo de projeto e experiência não pudessem interferir na análise dos resultados. 


\section{Resultados e Análises}

Serão apresentados primeiramente os dados da área de qualidade e depois as informações dos gerentes e líderes, e o resultado do uso do processo em 9 projetos. Para cada grupo serão apresentados os resultados do SPI e em seguida os do MBTI.

\section{1. Área de Qualidade}

O gráfico 1 apresenta os papéis de equipe mais relevantes identificados na área de qualidade: Gráfico 1: Resultado SPI da área de qualidade: 


\section{Gráfico 1: Resultado SPI da área de qualidade}

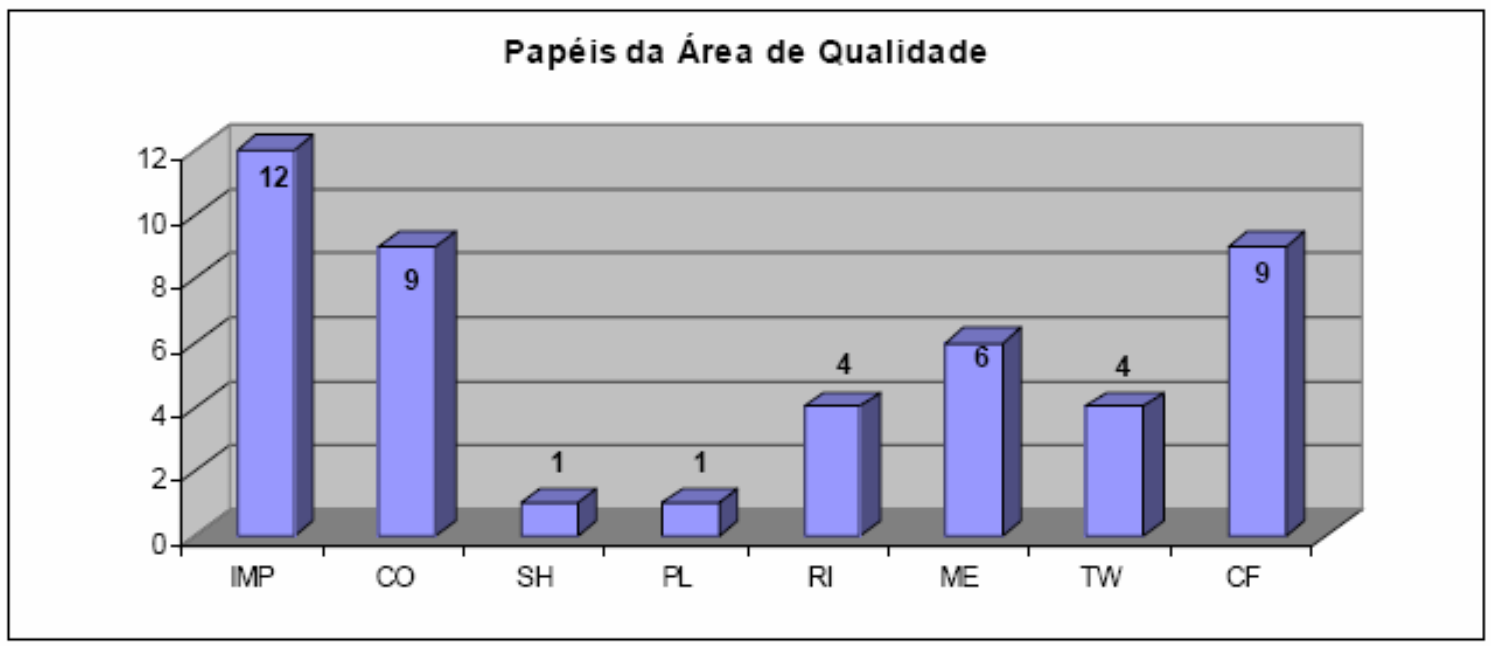

Os dados indicam a forte tendência da área de qualidade nos tipos Implementer (IMP), Completer Finisher (CF), Co-ordinator (CO) e Monitor Evaluator (ME). Percebe-se que há uma maior aglomeração de pessoas com o mesmo papel na área de qualidade com um perfil de controle, organização, críticos e, principalmente, atento aos detalhes. Este resultado é consistente com o trabalho de [Meira et al. 2008] que, utilizando outra abordagem metodológica, concluiu que os papéis em time de Belbin mais adequados para as funções de qualidade são $\mathrm{CO}$ e $\mathrm{CF}$, seguidos de ME e IMP.

Para o MBTI, dos 16 tipos psicológicos previstos, apenas 7 foram identificados na área de qualidade. $\mathrm{O}$ gráfico 2 apresenta os tipos psicológicos da equipe de qualidade:

Gráfico 2 : Tipos psicológicos do MBTI na área de qualidade

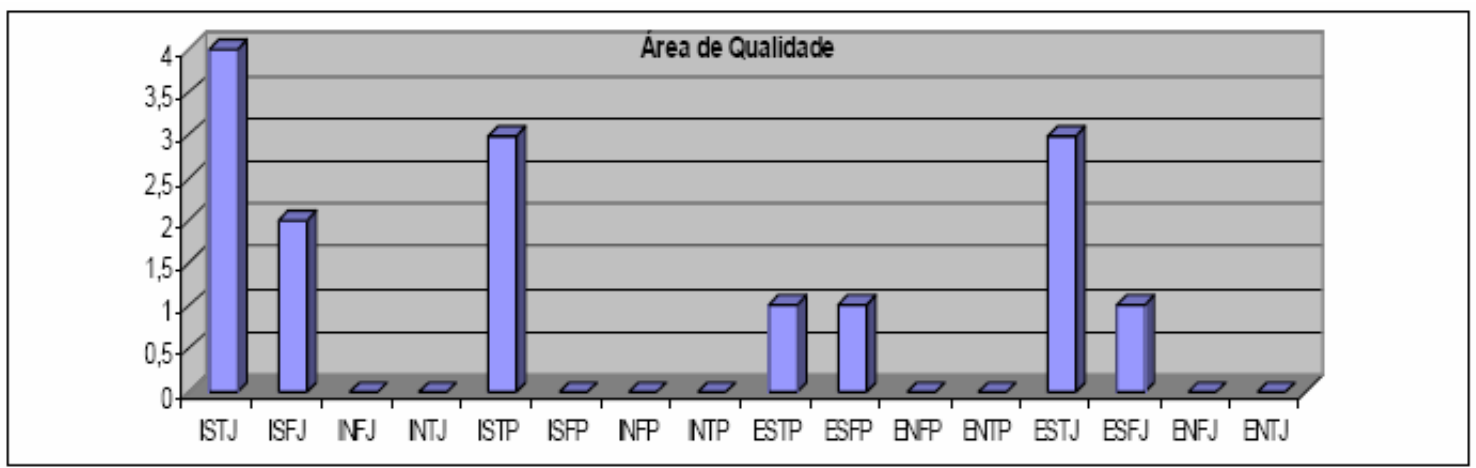

Nota-se que 8 pessoas possuem as letras STJ, e todas as outras possuem pelo menos uma combinação dessas letras, ou ST ou SJ, com exceção de 1 pessoa ESFP. Era esperado que o grupo de qualidade apresentasse uma tendência destes tipos psicológicos visto que as pessoas que atuam nesta área são mais favoráveis ao uso do processo. 


\subsection{Aderência dos Projetos ao Processo}

A tabela 2 apresenta a lista dos 9 projetos, e seus respectivos resultados nas áreas de planejamento (PP) e acompanhamento (PMC), e a relação quanto à duração e tamanho da equipe:

Tabela 2: Classificação dos projetos (auditorias PP e PMC, duração e equipe)

\begin{tabular}{|c|c|c|c|c|}
\hline Projeto & PP & PMC & Duração & Equipe \\
\hline 1 & A & A & G & M \\
\hline 2 & A & A & G & P \\
\hline 3 & A & A & M & M \\
\hline 4 & A & A & G & M \\
\hline 5 & A & A & G & M \\
\hline 6 & A & A & M & M \\
\hline 7 & I & A & G & P \\
\hline 8 & I & I & M & P \\
\hline 9 & I & A & G & P \\
\hline
\end{tabular}

Pode-se perceber inicialmente um padrão onde projetos de duração G e M com equipes de tamanho $\mathrm{M}$ utilizaram melhor o processo do que os projetos com equipes de tamanho P. Isso leva a crer que em equipes de tamanho $\mathrm{P}$ pode haver sobrecarga de trabalho nas atividades do projeto diminuindo as chances de utilização do processo. No caso de equipes de tamanho $M$ pode haver uma melhor distribuição das atividades e responsabilidades e maior disponibilidade das pessoas em utilizar o processo.

Dentre os projetos com equipe de tamanho $\mathrm{P}$ apenas o projeto 2 apresentou sucesso em ambos os processos. Nos projetos 7, 8 e 9 houve falha no processo de planejamento e / ou no de acompanhamento.

Pode-se inferir que um projeto $\mathrm{G}$ com equipe $\mathrm{M}$ precisa dos processos de $\mathrm{PP}$ e PMC mais formais e mais aderentes para apoiar as atividades de planejamento e acompanhamento do projeto. Já no caso de projetos com equipe $\mathrm{P}$, o processo pode ter sido subutilizado devido à equipe reduzida bem como sobrecarga de trabalho nas atividades do projeto.

\subsection{Avaliação dos Gerentes e Líderes dos Projetos}

A tabela 3 apresenta a lista dos 9 projetos, o resultado de aderência aos processos de PP e PMC, os papéis de equipe e tipos psicológicos identificados nos gerentes e líderes dos projetos: 
Tabela 3: Processos de PP e PMC versus SPI e MBTI Projeto

\begin{tabular}{|c|c|c|c|c|c|c|}
\hline Projeto & PP & PMC & Gerente SPI & Líderes SPI & $\begin{array}{c}\text { Gerente } \\
\text { MBTI }\end{array}$ & $\begin{array}{c}\text { Líderes } \\
\text { MBTI }\end{array}$ \\
\hline 1 & A & A & RI, PL & TW, CO, IMP, ME & ENFP & INFJ \\
\hline 2 & A & A & $\mathrm{CO}, \mathrm{TW}, \mathrm{IMP}$ & $\mathrm{CO}, \mathrm{ME}, \mathrm{CF}$ & ESFP & ISTJ \\
\hline \multirow{2}{*}{3} & \multirow{2}{*}{ A } & \multirow{2}{*}{ A } & \multirow{2}{*}{$\mathrm{SH}, \mathrm{PL}$} & $\mathrm{CF}, \mathrm{TW}, \mathrm{IMP}, \mathrm{ME}$ & \multirow{2}{*}{ ISTP } & ISTJ \\
\hline & & & & $\mathrm{CF}, \mathrm{SH}$ & & ESTJ \\
\hline \multirow{2}{*}{4} & \multirow{2}{*}{ A } & \multirow{2}{*}{ A } & \multirow{2}{*}{$\mathrm{ME}, \mathrm{CO}$} & IMP, ME, CO & \multirow{2}{*}{ ISFP } & ISTJ \\
\hline & & & & $\mathrm{CO}, \mathrm{CF}, \mathrm{ME}$ & & ISFJ \\
\hline \multirow{2}{*}{5} & \multirow{2}{*}{$\mathrm{A}$} & \multirow{2}{*}{$\mathrm{A}$} & \multirow{2}{*}{ RI, TW, CO } & CO, IMP, TW & \multirow{2}{*}{ ENFP } & ESTJ \\
\hline & & & & $\mathrm{CF}, \mathrm{SH}$ & & ENTP \\
\hline \multirow{3}{*}{6} & \multirow{3}{*}{ A } & \multirow{3}{*}{$\mathrm{A}$} & \multirow{4}{*}{$\mathrm{CO}, \mathrm{IMP}, \mathrm{CF}$} & IMP, CF, ME & \multirow{4}{*}{ ISFJ } & ESTJ \\
\hline & & & & $\mathrm{CF}, \mathrm{PL}$ & & ESTJ \\
\hline & & & & $\mathrm{ME}, \mathrm{PL}, \mathrm{CF}$ & & ESTJ \\
\hline 7 & I & $\mathrm{A}$ & & $\mathrm{CO}, \mathrm{IMP}$ & & ISTJ \\
\hline 8 & I & I & PL, ME & $\mathrm{ME}, \mathrm{CF}$ & INTP & ISTJ \\
\hline 9 & I & A & $\mathrm{ME}, \mathrm{RI}, \mathrm{CO}, \mathrm{SH}$ & $\mathrm{CF}$, IMP, CO & ESTJ & INTP \\
\hline
\end{tabular}

A partir da tabela 3, é possível perceber que todos os projetos, com exceção dos projetos 1 e 8, possuem um dos papéis de liderança indicados no SPI, no caso, o CO ou SH. Já os líderes apresentaram mais os papéis de IMP e CF que são papéis orientados à ação e execução.

Percebe-se que os projetos de 1 a 6 possuem uso adequado dos processos de PP e PMC, e neles existe pelo menos 4 papéis envolvidos, dos quais um deles é o papel de liderança $\mathrm{CO}$ ou $\mathrm{SH}$ e outro é um papel de ação $\mathrm{CF}$ ou IMP, e muitas vezes ambos os papéis. Isso leva a crer que a presença destes papéis dentro de uma equipe possibilitará o uso adequado dos processos de PP e PMC. Esses dados indicam o mesmo padrão identificado na área de qualidade, o que confirma e aumenta as evidências da predominância de alguns papéis favoráveis ao uso do processo.

Já os projetos que apresentaram resultado insatisfatório, no caso os projetos 7, 8 e 9 foram cuidadosamente avaliados para confirmar ou acrescentar novas informações ao resultado anterior.

Podemos perceber que os projetos 7 e 8 contêm o menor número de papéis envolvidos, apenas 3 . O projeto 7 apesar de possuir um dos papéis de liderança e um dos 
papéis orientados à ação, obteve resultado insatisfatório apenas no processo de PP. Podemos considerar que a ausência do papel SH no gerente ou no líder prejudicou o uso do processo de PP. Entretanto, se considerarmos os dados da tabela 2, identificamos que o projeto 7 tem uma equipe pequena, o que leva a crer que o gerente e o líder ficaram sobrecarregados na fase inicial do projeto e por sua vez, não conseguiram cumprir com as atividades previstas no processo, mas que, conseqüentemente, levou a uma maior necessidade de acompanhamento do projeto.

Já no caso do projeto 8 , o resultado foi insatisfatório em ambos os processos, tanto PP como PMC. Este projeto também contém o menor número de papéis envolvidos, só que neste caso não possui nenhum dos papéis de liderança, e possui apenas um dos papéis orientados à ação. Considerando a tabela 2, podemos verificar que é um projeto de duração $\mathrm{M}$ e equipe de tamanho $\mathrm{P}$, o que agrava ainda mais a situação do não uso do processo. Isso leva a crer que o gerente juntamente com o líder estavam focados em executar as atividades do projeto, visto que o período era médio, e não houve disponibilidade em executar as atividades do processo. Além disso, podemos considerar que a ausência dos papéis $\mathrm{CO}, \mathrm{SH}$ e IMP prejudicaram o uso do processo. Este resultado reforça a importância de um papel de liderança na condução dos processos de PP e PMC.

$\mathrm{Na}$ análise do projeto 9 , podemos chegar a mesma conclusão do projeto 7. Apesar de existirem 6 papéis de Belbin, dos quais há a presença de um papel de liderança e um papel orientado à ação, podemos alinhar ao resultado da tabela 2, que apresenta o projeto 9 com uma equipe pequena, o que pode ter levado a uma sobrecarga dos gerentes e líderes e falta de uso do processo, mas com maior necessidade de acompanhamento posterior.

Além dos papéis do SPI, é possível identificar um padrão nos tipos psicológicos do MBTI nos líderes de projeto. A partir da tabela 2, é possível perceber que todos os projetos, com exceção do projeto 1, possuem o tipo psicológico ISTJ ou ESTJ. A maioria destes tipos foi identificada nos líderes de projetos, sendo apresentado em apenas um gerente. Apesar dos gerentes serem mais perceptivos (letra P), eles possuem líderes com características correspondentes às identificadas na área de qualidade, que indica o tipo STJ mais favorável ao uso do processo.

Para o MBTI, a análise deve considerar não apenas a combinação dos tipos psicológicos entre gerentes e líderes, mas também a combinação das letras de cada tipo e do índice de clareza de cada uma delas.

É possível perceber que dos projetos 1 a 6 que tiverem resultado adequado em ambos os processos de PP e PMC, 5 gerentes possuem a letra $\mathrm{P}$ em seu tipo psicológico, indicando pessoas perceptivas o que indica pessoas espontâneas e flexíveis. Este resultado leva a crer que este tipo é menos resistente à utilização de processos visto que são pessoas abertas. Estes mesmos projetos possuem líderes com a letra J, que são pessoas organizadas e planejadas. Logo, podemos inferir que o gerente fica aberto ao uso do processo e tem o apoio do líder para executá-lo.

O projeto 7 é um caso particular, visto que o mesmo possui um gerente com a letra $\mathrm{F}$, mas não tem a letra $\mathrm{P}$, e um líder com a letra $\mathrm{J}$, e houve insatisfatoriedade no processo de 
PP. Além da ausência da letra $\mathrm{P}$ e, de acordo com o quadro 18, o projeto 7 tem duração $\mathrm{G}$ e uma equipe $\mathrm{P}$, o que leva a crer que o esforço inicial para organizar o projeto impediu a utilização adequada do uso do processo de PP, visto que o acompanhamento foi executado de forma adequada.

No caso do projeto 8 temos um gerente atípico com um perfil que envolve a combinação das letras NTP, o que indica uma pessoa intuitiva, lógica, objetiva, espontânea e flexível. Apesar de ter a letra $\mathrm{P}$, não existe a combinação $\mathrm{FP}$, podemos considerar que a análise do resultado SPI indica a ausência de um dos papéis de liderança, o que levar a crer que isso prejudicou o uso do processo.

Já o projeto 9 , é similar à análise do projeto 7 , visto que também é um projeto grande com equipe pequena, e pode ter sofrido demanda de planejamento no início do projeto prejudicando as atividades do processo.

De todos os projetos apresentados, o projeto 8 confirma que a ausência da combinação das letras FP no tipo psicológico do gerente prejudica a utilização do processo, visto que a dicotomia TJ para o caso do gerente pode ser mais resistente a aceitar o seguimento de padrões. Consideramos que as letras FP devem estar presentes no gerente de projeto, combinada com líderes que possuem as letras STJ para garantir que os processos de PP e PMC sejam utilizados.

Apesar da pequena amostra e do seu caráter qualitativo desta pesquisa, é possível observar que há uma relação entre o papel de equipe e tipos psicológicos dos gerentes e líderes de projetos na utilização de processos, e que os mesmos estão alinhados à outras pesquisas na área de qualidade na qual foram identificados os mesmos papéis e tipos predispostos ao uso do processo, no caso CO, CF, IMP, e tipos STJ.

Mais especificamente, foi possível identificar que a área de qualidade possui um padrão do papel de equipe bem como tipo psicológico que corresponde às características de pessoas organizadas, planejadas, críticas, sistemáticas e que contribuem para o uso do processo. Portanto, esta pesquisa confirma a hipótese de que existe uma tendência natural de pessoas com estes perfis a se interessarem pela área de qualidade e pelo uso do processo.

Além do comportamento do gerente e líder, fica explícito que a duração do projeto atrelada ao tamanho da equipe, conforme citado por Gorla e Lam (2004), são variáveis que devem ser consideradas durante a composição da equipe, visto que ainda que os gerentes e líderes tenham os perfis indicados, essas variáveis podem prejudicar a utilização dos processos de PP e PMC.

\section{Considerações Finais}

O motivo principal para conhecer os tipos de personalidade é permitir que o processo possa ser orientado às pessoas, desde a sua forma de escrita, os treinamentos realizados, às ferramentas e atividades. É possível identificar aquelas pessoas que são naturalmente aptas ao uso do processo e aqueles que não são favoráveis ao uso do processo.

A pesquisa apresentou a similaridade entre os papéis de equipe e tipos psicológicos 
da área de qualidade e gerentes e líderes de projetos no sucesso do uso dos processos de PP e PMC, e mostra que existe uma característica comum nas pessoas que estão em busca da qualidade. É necessário a inserção dessas pessoas dentro das equipes, ou permitir que equipes sejam formadas garantindo a participação de pessoas com papéis $\mathrm{CO}, \mathrm{CF}$ e IMP e tipo psicológico com as letras STJ para maximizar a utilização dos processos nos projetos.

Os dados apresentados refletem que há uma tendência de uso do processo por um certo tipo de indivíduo, seja pelo seu papel de equipe ou pelo seu tipo psicológico, ou a combinação destes, e que seu comportamento influencia a equipe no uso do processo. Foi possível observar que a duração do projeto e o tamanho da equipe também influenciam no uso do processo.

Este trabalho teve como objetivo relacionar a influência dos gerentes e líderes de projeto, e área de qualidade, e seus respectivos papéis de equipe e tipos psicológicos, no uso de processos de desenvolvimento de software. Verificou-se que fatores humanos e sociais afetam o uso do processo.

Por fim, vale ressaltar a importância de estudar cada vez mais aspectos humanos e aplicabilidade das teorias de personalidade dentro da engenharia de software, possibilitando estender o conhecimento destas teorias ainda pouco utilizadas no Brasil.

\section{Agradecimentos}

Os autores agradecem a revisão realizada pela doutora Renata Gonçalves Ferreira, pesquisadora da Pós-Graduação em Psicobiologia da Universidade Federal do Rio Grande do Norte.

\section{Referências}

Aritzeta, A. ; Senior, B. ; Swailes, S. (2005) Team role preference and cognitive styles: a convergent validity study. Small Group Research, (S.1.: s.n.), v. 36, n. 4, p. 404- 435.

Belbin, M. R. (1981) Management Teams: Why they succeed or Fail? ButterworthHeinemann Ltd, ISBN 0750626763.

. (1993) Team Roles at Work. At Linarc House, Jordan Hill, Oxford: ButterworthHeinemann, ISBN 0750626755.

Cunha, A. D. and Greathead, D. (2007) Does personality matter? : an analysis of codereview ability. Communications of the ACM. New York, USA : Associatiom for Computing Machinery, v. 50, n. 5, p. 109-112, Maio.

Fernandes, F. e Silva, F. (2007) Relações entre competências pessoais e tipos de personalidade do gerente de projetos. In: Congresso Brasileiro em Gerenciamento de Projetos, 2., 2007, Salvador-BA-Brasil. Resumos.... Project Management Institute Chapter Bahia,.

França, C. A. e Silva. F. Q. B. (2007) Um estudo sobre relações entre papéis funcionais do RUP e o comportamento pessoal no trabalho em equipe em fábricas de software. In: 
WOSES - Workshop: um olhar sociotécnico sobre a engenharia de software, 3., 2007, Ipojuca/PE. Resumos.... Sociedade Brasileira de Computação.

Gorla, N. and Lam, Y.W. (2004) Who should work with whom? Building effective software project teams. Communication of the ACM, June, v. 47, n. 6.

Henry, S. M. and Stevens, K. T. (1999) Using Belbin's Leadership Role to Improve Team Effectiveness: an empirical investigation, Journal of Systems and Software, v. 44, n. 3, Jan., p. 241-250.

Meira, A.F, et al. (2008) Um estudo da adequação de perfis profissionais para o SQA em empresas de desenvolvimento de software, Relatório de Pesquisa, UFPE, Recife/PE.

Mendes, F. F. ; Oliveira, J. L. ; Fernandes, P. G. ; Souza, A. (2007) Análise de Riscos na Implantação de Melhorias de Processos de Software. ProQuality (UFLA), v. 3, p. 25 32.

Myers, I. and Briggs, K. Myers-Briggs Type Indicator. Disponível em: <http://www.myersbriggs.org/>. Acesso em: 26 set. 2007.

Pereira, M. S. (2005) Implicações Práticas da gerência de projetos baseada em aptidões dos membros. Trabalho de graduação em empreendedorismo, UFPE, 2005.

Rocha, A.R. et al. (2005) Dificuldades e Fatores de Sucesso na Implantação de Processos de Software Utilizando o MR-MPS e o CMMI, In: Workshop de Implementadores MPS.BR, 1. Resumos... Brasília, Brasil. Out.

Roullier, A. C. et al. (2006) Metodologias e analise das implantacoes do MPS-BR realizadas pela SWQuality. Revista ProQuality: Qualidade na Producao de Software. v. 2, n. 2, p. 57-63.

Sharp, H. and Robinson, H. (2005) Some Social Factors of Software Engineering: the maverick, community and technical practices, Human and Social Factors of Software Engineering (HSSE), May 16.

Souza, A. S.; Oliveira, J. L.; Jino, M. (2004) Experiências e riscos na implantação de processos de software em empresas do centro-oeste brasileiro. In de Ingeniería del Software e Ingeniería del Conocimiento, Q. J. I., editor, Anais.... Quarta Jornadas IberoAmericanas de Ingeniería del Software e Ingeniería del Conocimiento, volume 1, pages 1-4, Madri.

Stevens, T. K. (1998) The Effects of Roles and Personality Characteristics on Software Development Team Effectiveness, Virginia Polytechnic Institute and State University.

Vasconcelos, A. M. L.; Rouiller, A.C.; Machado, C. Â. F.; Medeiros, T. M. M. (2006) Introdução à Engenharia de Software e à Qualidade de Software. Lavras: UFLA/FAEPE. 157 p. :il. - (Curso de Pós- graduação "Lato Sensu" (Especialização) à Distância - Melhoria de Processo de Software. 